\title{
Treatment of symptomatic uterine fibroids with green tea extract: a pilot randomized controlled clinical study
}

This article was published in the following Dove Press journal:

International Journal of Women's Health

6 August 2013

Number of times this article has been viewed

\author{
Eman Roshdy 1 ,* \\ Veera Rajaratnam ${ }^{2, *}$ \\ Sarbani Maitra ${ }^{2}$ \\ Mohamed Sabry 3,4 \\ Abdou S Ait Allah ${ }^{3,4}$ \\ Ayman Al-Hendy ${ }^{2}$ \\ 'Department of Public Health \\ and Community Medicine, Sohag \\ University, Sohag, Egypt; ${ }^{2}$ Department \\ of Obstetrics and Gynecology, \\ Meharry Medical College, Nashville, \\ TN, USA; ${ }^{3}$ Department of Obstetrics \\ and Gynecology, Sohag University, \\ Sohag, Egypt; ${ }^{4}$ Ibn Sina IVF Center, \\ Ibn Sina Hospital, Sohag, Egypt \\ *These authors contributed equally \\ to this work
}

Correspondence: Ayman Al-Hendy Department of Obstetrics and Gynecology, Center for Women's Health Research, Meharry Medical College, 1005 Dr DB Todd Jr Boulevard, Nashville, TN 37208, USA

$\mathrm{Tel}+\mathrm{I} 6159633107$

$\mathrm{Fax}+16159633125$

Email ahendy@mmc.edu
Background: Uterine fibroids (UFs, also known as leiomyoma) affect $70 \%$ of reproductive-age women. Imposing a major burden on health-related quality-of-life (HRQL) of premenopausal women, UF is a public health concern. There are no effective medicinal treatment options currently available for women with symptomatic UF.

Objectives: To evaluate the efficacy and safety of green tea extract (epigallocatechin gallate [EGCG]) on UF burden and quality of life in women with symptomatic UF, in a double-blinded, placebo-controlled randomized clinical trial.

Methods: A total of 39 reproductive-age women (age 18-50 years, day 3 serum folliclestimulating hormone $<10 \mathrm{mIU} / \mathrm{mL}$ ) with symptomatic UF were recruited for this study. All subjects had at least one fibroid lesion $2 \mathrm{~cm}^{3}$ or larger, as confirmed by transvaginal ultrasonography. The subjects were randomized to oral daily treatment with either $800 \mathrm{mg}$ of green tea extract (45\% EGCG) or placebo ( $800 \mathrm{mg}$ of brown rice) for 4 months, and UF volumes were measured at the end, also by transvaginal ultrasonography. The fibroid-specific symptom severity and HRQL of these UF patients were scored at each monthly visit, using the symptom severity and quality-of-life questionnaires. Student's $t$-test was used to evaluate statistical significance of treatment effect between the two groups.

Results: Of the final 39 women recruited for the study, 33 were compliant and completed all five visits of the study. In the placebo group $(n=11)$, fibroid volume increased $(24.3 \%)$ over the study period; however, patients randomized to green tea extract ( $\mathrm{n}=22,800 \mathrm{mg}$ /day) treatment showed significant reduction $(32.6 \%, P=0.0001)$ in total UF volume. In addition, EGCG treatment significantly reduced fibroid-specific symptom severity $(32.4 \%, P=0.0001)$ and induced significant improvement in HRQL $(18.53 \%, P=0.01)$ compared to the placebo group. Anemia also significantly improved by $0.7 \mathrm{~g} / \mathrm{dL}(P=0.02)$ in the EGCG treatment group, while average blood loss significantly decreased from $71 \mathrm{~mL} / \mathrm{month}$ to $45 \mathrm{~mL} / \mathrm{month}$ $(P=0.001)$. No adverse effects, endometrial hyperplasia, or other endometrial pathology were observed in either group.

Conclusion: EGCG shows promise as a safe and effective therapeutic agent for women with symptomatic UFs. Such a simple, inexpensive, and orally administered therapy can improve women's health globally.

Keywords: EGCG, leiomyoma, uterine fibroid, green tea, quality of life, pilot clinical study

\section{Introduction}

Uterine fibroids (UFs also known as uterine leiomyoma) are very common, benign estrogen-dependent tumors of the uterine wall, ${ }^{1}$ affecting up to $50 \%$ of women aged 35-49 years. ${ }^{2}$ UFs cause multiple clinical complications, such as acute and chronic pelvic pain, excessive vaginal bleeding, dyspareunia, and iron-deficiency anemia, 
as well as miscarriage and infertility. ${ }^{3}$ The impact of these serious clinical complications on women's health is tremendous. ${ }^{1}$ Being more prevalent in African-American women, uterine leiomyoma is a major health-disparity issue further diminishing quality of life, especially for women of color, who are affected by this disease. ${ }^{4}$ Currently, there is no effective long-term medicinal treatment for this common debilitating disease. Gonadotropin-releasing hormone (GnRH) analogues are effective short-term medical therapy options, but FDA-approved only as preoperative adjuvant therapy (3 months or less) because of the risk of irreversible bone loss and osteoporosis and other major side effects. ${ }^{1}$ The current management options range from hysterectomy (removal of the uterus), myomectomy, uterine artery embolization, or image-guided focused ultrasound thermal therapy, to observation (in mild cases). ${ }^{3,5,6}$ Surgery, usually in the form of hysterectomy, is the traditional treatment option for women with symptomatic UFs. This radical option creates a major dilemma for women with symptomatic UFs who have not completed their families or are yet to start one. Problems with fibroids must be viewed as a public health concern because of their major burden on women's reproductive health, in general, and on minority women's health, in particular. We and others are exploring potential noninvasive treatment options for uterine leiomyoma, such as localized gene therapy, antiprogesterones, vitamin D, and green tea-extract supplements. ${ }^{5-11}$

Green tea is a natural product, commonly used by women for multiple purposes. Epigallocatechin gallate (EGCG), the major catechin in green tea, exhibits several useful biological effects, including anti-inflammatory, antiproliferative, and antioxidant effects. A study conducted by the US Department of Agriculture reported that green tea has potent anticancer effects against a wide range of human cancer cells. ${ }^{12}$ Green tea's polyphenols are considered responsible for these positive effects, and most notably EGCG has been shown to inhibit key pathways of tumor growth. ${ }^{13,14}$ Several human clinical studies used EGCG for extended periods of time in which EGCG was very well tolerated with virtually no reportable side effects. Wolfram ${ }^{14}$ used $300 \mathrm{mg} /$ day oral EGCG in patients with cardiac disease, and reported improved endothelial function and increased brachial artery flowmediated dilation that paralleled changes in EGCG plasma concentration. In another study, Ahn et al investigated the clinical efficacy of green tea extracts (EGCG) delivered in the form of a capsule in patients with human papilloma virus-infected cervical lesions. Patients with cervical lesions (chronic cervicitis, mild dysplasia, moderate dysplasia, and severe dysplasia) were divided into different groups receiving oral EGCG (200 mg/day for 8-12 weeks) with or without a local ointment containing EGCG and were compared with untreated patients as control. In their study, $60 \%$ of patients (six of ten) undergoing EGCG capsule therapy showed a response compared to a $10 \%$ response rate (four of 39 ) in untreated controls $(P<0.05)$. The effectiveness of green tea extracts in a capsule form for the treatment of cervical lesions suggests that oral intake of green tea extracts can be a potential systemic therapy regimen. ${ }^{15}$

Numerous studies have described favorable effects of oral EGCG therapy when used in overweight and obese individuals for extended durations. Chow et a ${ }^{16}$ reported on human use of EGCG in 30 healthy volunteers randomly assigned to one of the following doses of polyphenol E (a decaffeinated green tea catechin mixture): 400, 800, or $1200 \mathrm{mg}$ /day (ten subjects per dose group). Only transient mild nausea was noted in 3\% of the study participants, and only at the highest study agent dose (1200 mg EGCG/day) and in fasting state. Luo et al reported on the consumption of 500-1000 mg/day of oral EGCG for 3 months, which resulted in marked decreases in several oxidative biomarkers compared to placebo. ${ }^{17}$ Doses of $800 \mathrm{mg}$ were well tolerated, with no reported side effects. ${ }^{16,17}$ Recent studies from our laboratory demonstrated the ability of EGCG to inhibit proliferation and to induce apoptosis in human leiomyoma cells in animal and in vitro models. These preclinical data in a mouse model with preexisting fibroid lesions demonstrated that EGCG at a dose of $1.25 \mathrm{mg} / \mathrm{kg} /$ day added to drinking water was able to induce a highly significant (up to $80 \%$ ) shrinkage of fibroid lesions compared to untreated controls. ${ }^{11,18}$ The treatment was well tolerated, with no detected side effects. Such daily doses of EGCG have also been administered safely to human subjects in prior clinical trials. This shrinkage of fibroids is partially attributed to potent inhibitory effects of EGCG on catechol- $O$-methyltransferase (COMT), an enzyme that we have recently linked to the pathogenesis of UFs. ${ }^{19}$ We have recently demonstrated elevated COMT levels in UFs compared to adjacent myometrium, and described the important role of COMT in fibroid pathogenesis. Interestingly, recent reports demonstrated that EGCG has potent anti-COMT activity, ${ }^{20-22}$ and one of these reports has also shown that green tea-extract intake is associated with weight loss due to EGCG-induced thermogenesis. EGCG is known to be capable of inhibiting COMT - the enzyme that degrades norepinephrine - thus resulting in the accumulation of norepinephrine that stimulates thermogenesis. The inhibition of COMT by EGCG 
was uncompetitive with respect to $S$-adenosylmethionine, suggesting that there is no direct interaction between EGCG and the $S$-adenosylmethionine-binding site on COMT, yet there was clear inhibition of COMT activity with increasing EGCG concentration. Our preliminary data using human leiomyoma cells showed that EGCG also inhibited COMT gene expression in a dose-dependent manner. ${ }^{23}$

Armed with our preclinical findings, in this pilot doubleblinded placebo-controlled clinical trial, we set out to evaluate the efficacy and safety of oral green tea extract (EGCG) in women with symptomatic UFs. The primary aim of this study was to test the hypothesis that oral green tea extract, at $800 \mathrm{mg}$ (45\% EGCG) daily for 4 months, could reduce UF size. Secondary aims were to assess the effect of green tea extract (EGCG) on leiomyoma-specific symptom severity (SS) and health related quality-of-life (HRQL) among women with symptomatic leiomyoma, utilizing the validated UF symptom quality-of-life questionnaire (UFS-QOL). ${ }^{24}$

Briefly, this pilot double-blinded placebo-controlled clinical trial was designed to evaluate the efficacy and safety of oral green tea extract (EGCG) treatment in reproductive-age women with symptomatic UFs.

\section{Materials and methods Subjects}

Women were eligible for inclusion in the trial if they were 18 years of age or older, were premenopausal with folliclestimulating hormone (FSH) level less than $10 \mathrm{mIU} / \mathrm{L}$, had reported at least moderately severe leiomyoma-related symptoms (a score of $\geq 25$ on the UF quality-of-life symptom severity subscale), had a total uterine volume of $\geq 160 \mathrm{~mL}$ by vaginal and abdominal ultrasound and at least one UF/ leiomyoma that was $\geq 2 \mathrm{~cm}^{3}$. Women were excluded if they were pregnant or intended to become pregnant during the next 6 months, were currently breastfeeding, had an untreated abnormal pap smear, or had major medical morbidity or severe anemia with hemoglobin $<8.0 \mathrm{mg} / \mathrm{dL}$, elevated liver enzymes more than 1.5 times the upper limit of normal, or active substance abuse. In addition, women were excluded if they reported current or recent (within the past 3 months) use of the following medications: oral or systemic corticosteroids, hormones (estrogen, progestin, oral contraceptives), herbal or botanical supplements with possible hormonal or EGCG effects, or if they reported use of GnRH analogues or Depo-Provera within the past 6 months. All study participants agreed to use barrier contraception and not use hormonal treatments for leiomyoma during the course of the trial.

\section{Study methodology}

This study was conducted at the Department of Obstetrics and Gynecology, Faculty of Medicine, Sohag University, and the Ibn Sina IVF Center, Sohag, Egypt. The study protocol was approved by the ethical committee of the Faculty of Medicine, Sohag University. The period of enrollment and recruitment of the participants was from November 2010 to August 2011. All participants $(n=33)$ were Egyptian women, and informed consent was obtained from them prior to any study procedures. The study pharmacist at Sohag Faculty of Medicine carried out the randomization process (by sequential digital assignment coding) and dispensed green tea extract or placebo capsules to participants, based on the assignment code randomly picked by each participant prior to treatment. The assignment code key was kept in the possession of the pharmacist until the study was complete. The study staff and the participants were blinded to the treatment assignment throughout the whole study. Both the green tea extract and placebo capsules were purchased from Beehive Botanicals (Hayward, WI, USA), but this company did not fund the study. Green tea-extract capsules were $400 \mathrm{mg}$ in weight, and each capsule contained 95\% polyphenols by ultraviolet detection and 45\% EGCG by high-performance liquid chromatography. The placebo capsules were identical to the EGCG capsules in appearance and weight, and contained $400 \mathrm{mg}$ of inert material (brown rice). The women participants were thus randomly assigned to the two arms of this 4-month study: daily dosage of two capsules of green tea ( $800 \mathrm{mg} /$ day) or two capsules of placebo. The capsules were taken after meals to avoid possible irritation to stomach mucosa.

Protocol adherence was determined from monthly logs and counts of returned pills. Outcomes were assessed using validated measures. The primary outcome was the mean change in uterine leiomyoma burden (volume and number), which was assessed by transvaginal ultrasonography (TVU), and/or transabdominal ultrasonography, at baseline (visit 1) and at the end of the 4-month (visit 5) treatment period. The visit 1 procedure consisted of a brief history and physical examination, including biometric measurement. The uterus was measured in three planes, and a total volume was calculated. All visible UFs were identified, numbered, and the total fibroid volume calculated through measuring the length, width, and height, then using the formula $(0.532 \times$ length $\times$ width $\times$ height $)$ for each of the fibroids, and the results summed (total volume). The secondary measure done at every visit was the mean change in fibroidspecific health-related quality of life (UFS-QOL), ${ }^{24}$ which 
has two scales: the first assesses SS, with a scale range from 5 to 40, where high values are indicative of greater symptom severity. A sample question from this scale is: "During the past month, how distressed were you by heavy bleeding during your menstrual period, feeling tightness or pressure in your pelvic area, or feeling fatigued?" The second scale is the HRQL questionnaire, which measures perceived impact of leiomyoma on activities of daily living, general concern and worry, energy, mood, sense of self-control, selfconsciousness, and sexual functioning of the participants. The scale for this second questionnaire ranges from 29 to 145; the higher the scores, the better the quality of life. Blood loss was also assessed monthly by means of a daily menstrual log, with visual assessment of quantity of blood lost using menstrual pictogram. The scores (in milliliters) were associated with each icon given in a pictogram ${ }^{25}$ that was provided to all participants. Each of these questionnaires was assessed monthly. Safety monitoring included monthly hemoglobin levels, liver- and kidney-function tests, and pregnancy testing. Because of our recently reported effects of inflammation on $\mathrm{UFs}^{26}$ and possible anti-inflammatory effects of EGCG, ${ }^{27,28}$ we assessed specific markers of inflammation in all participants. We measured the levels of two serum inflammation biomarkers (interleukin 6 and tumor necrosis factor- $\alpha)^{29,30}$ and one urinary biomarker (isoprostane) $)^{31}$ of inflammation. All participants underwent endometrial biopsy at baseline (visit 1) and at the end of the study after 4 months (visit 5 ) to confirm the presence of normal endometrium and to ensure the safety of EGCG treatment on endometrium histology. ${ }^{32,33}$ To follow up on possible adverse events, a monthly data safety-monitoring meeting was held to review the lab results and monitor the study participants' compliance and adverse effects.

\section{Statistical analysis}

Data entry, screening, and analysis were carried out by the authors using SAS version 9.0 (SAS Institute, Cary, NC, USA). Descriptive analysis was done using mean, frequency, standard deviation, and percentage. Student's $t$-test was used to compare and evaluate statistical significance of treatment effect between groups. A $P$-value of 0.05 was considered significant.

\section{Results}

\section{Characteristics of study participants}

Despite extensive advertisement and with all efforts invested, only 39 of 72 age-eligible women consented during the enrollment phase. Of these, 22 were randomized to receive green tea extract and 17 to receive placebo (Figure 1). The baseline characteristics of the two groups were well matched, with no significant difference (Table 1). The dropout rate was low: only six women from the placebo group dropped out after randomization and before any additional follow-up measures could be obtained. Compliance was high among women in both the treatment $(n=22)$ and the placebo groups $(n=11)$. Subjects reported missing a maximum of three doses per month.

\section{Effect of green tea extract (EGCG) treatment on total uterine fibroid volume in patients}

The total fibroid volumes were measured by TVU at visit 1 and at visit 5 for both treatment groups. For each patient, the change in total fibroid volume at the end of the treatment period was calculated as a percentage of visit 1 volume. At the end of 4 months of treatment, the mean total fibroid volume dramatically decreased by an average of $32.6 \%$ in the EGCG group (Figure 2), while the placebo group showed an average increase of $24.2 \%$ (Figure 2 ). The overall percentage reduction in mean total fibroid volume in the EGCG-treated group was highly significant (Figure 2, $P<0.0001$ ) compared to the placebo group.

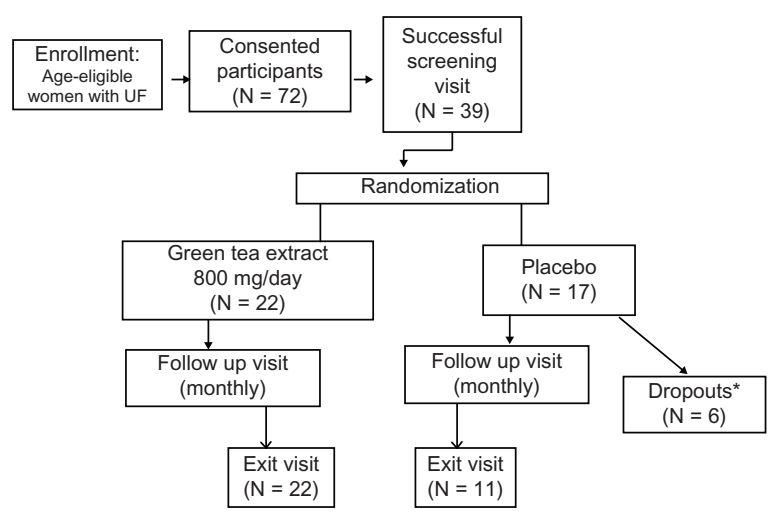

Figure I Flowchart of participant recruitment and flow through randomized clinical epigallocatechin gallate (EGCG) treatment study. Only 39 of the 72 age eligible women consented during the enrollment phase. The ethical committee of the Faculty of Medicine, Sohag University approved the protocol. All consenting participants $(n=39)$ were confirmed by transvaginal ultrasonography to have uterine fibroids and randomized to treatment groups: 22 were randomized to receive green tea extract (EGCG, oral dose, $800 \mathrm{mg} /$ day) and 17 to receive placebo capsules. The baseline characteristics of the two groups were well matched, with no significant difference (Table I). After randomization and before any additional follow-up measures could be obtained, six patients dropped out of the placebo group. Compliance throughout the 4-month treatment period (visit I to visit 5) was high among the remaining patients in both treatment groups: EGCG $(n=22)$ and the placebo group $(n=I I)$. Uterine fibroid volumes were measured again by transvaginal ultrasonography at the end of the 4-month treatment period (visit 5). * Signifies patients that dropped out after randomization.

Abbreviation: UF, uterine fibroids. 
Table I Baseline characteristics of the study population

\begin{tabular}{|c|c|c|c|c|c|c|c|}
\hline \multirow[t]{2}{*}{ Subjects } & \multicolumn{3}{|c|}{$\begin{array}{l}\text { Treatment group } \\
(\mathrm{n}=22)\end{array}$} & \multicolumn{3}{|c|}{$\begin{array}{l}\text { Placebo group } \\
(n=I I)\end{array}$} & \multirow[t]{2}{*}{$P$-value } \\
\hline & Mean (SD) & Median & Range & Mean (SD) & Median & Range & \\
\hline Age (years) & $4 I .5(5.9)$ & 42 & $25-50$ & $43(6.6)$ & 44 & $35-52$ & $0.5 \mathrm{I}$ (NS) \\
\hline Parity & $\mathrm{I} .04(\mathrm{I} .4)$ & 1 & $0-5$ & $1.09(1.4)$ & 1 & $0-4$ & 0.94 (NS) \\
\hline Gravidity & $1.68(1.7)$ & 2 & $0-7$ & $\mathrm{I} .4(\mathrm{I} .8)$ & 1 & $0-5$ & 0.60 (NS) \\
\hline BMI $\left(k g / m^{2}\right)$ & $33(6.9)$ & 33.5 & $21.9-48.4$ & $32.2(8.2)$ & 31.2 & $20.8-45.3$ & 0.73 (NS) \\
\hline Fibroid total volume change* & \multicolumn{3}{|l|}{$32.6 \%$ (decrease) } & \multicolumn{3}{|c|}{$24.3 \%$ (increase) } & $<0.0001 * *$ \\
\hline
\end{tabular}

Notes: The baseline characteristics (age and BMI) of the study patients randomized to the two treatment (EGCG vs placebo) groups were well matched, with no significant difference. A $P$-value of $\leq 0.05$ was considered statistically significant. *Visit 5 volume as percentage of volume at visit I; **significant difference.

Abbreviations: BMI, body mass index; EGCG, epigallocatechin gallate; SD, standard deviation; NS, not significant.

\section{Effect of green tea extract (EGCG) treatment on fibroid-specific symptom severity in patients with uterine fibroids}

The mean SS percentile score dramatically decreased in patients with 4 months' (visit 5 score $<$ visit 1 score) use of green tea extract (EGCG, Figure 3) while the mean SS percentile score increased in patients randomized to the placebo

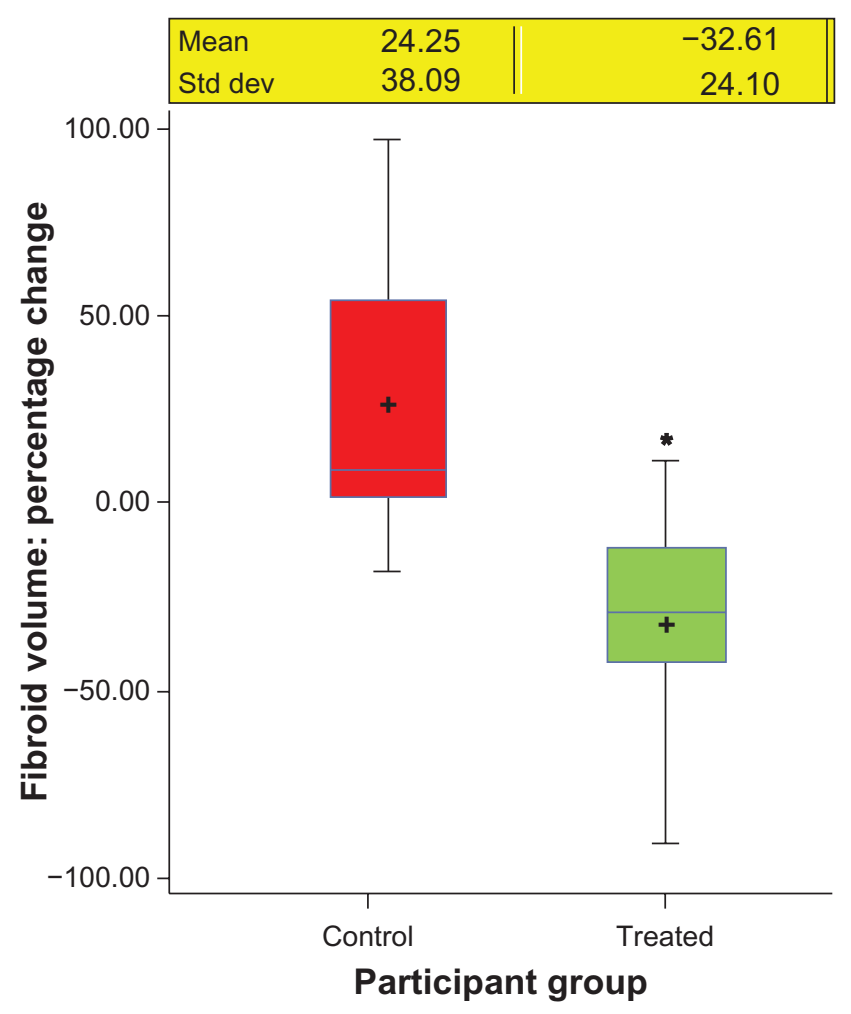

Figure 2 Epigallocatechin gallate (EGCG) treatment decreases total uterine fibroid volume in patients. Fibroid volume decreased significantly with 4 months' (visit 5 score < visit I score) use of green tea extract (EGCG, oral dose, $800 \mathrm{mg} /$ day). Fibroid volumes were measured by vaginal and abdominal ultrasonography. The mean change in fibroid volume for the EGCG-treated group was $=-32.6 \mathrm{I}( \pm 24 . \mathrm{I})$, a significant decrease $(t=5.25 ; P<0.0001)$ compared to the placebo group, for which mean fibroid volume increased by 24.25 ( \pm 38.09$)$. A $P$-value of $\leq 0.05$ was considered statistically significant and is indicated by the asterisk. The box plot shows the maximum and minimum values as well as the mean and the median. Mean values are indicated by the plus signs. group. The mean change in SS score for the EGCG-treated group was $-25.28( \pm 17.38$, ) and significantly low $(t=5.22$, $P<0.0001)$ compared to the placebo group, for which mean change in SS score was $=7.1( \pm 15.5)$.

\section{Effect of green tea-extract treatment on fibroid-specific health-related quality of life in patients with uterine fibroids}

Leiomyoma-specific quality-of-life measures were similar between both groups at baseline (visit 1). The EGCG-treated group demonstrated an overall average

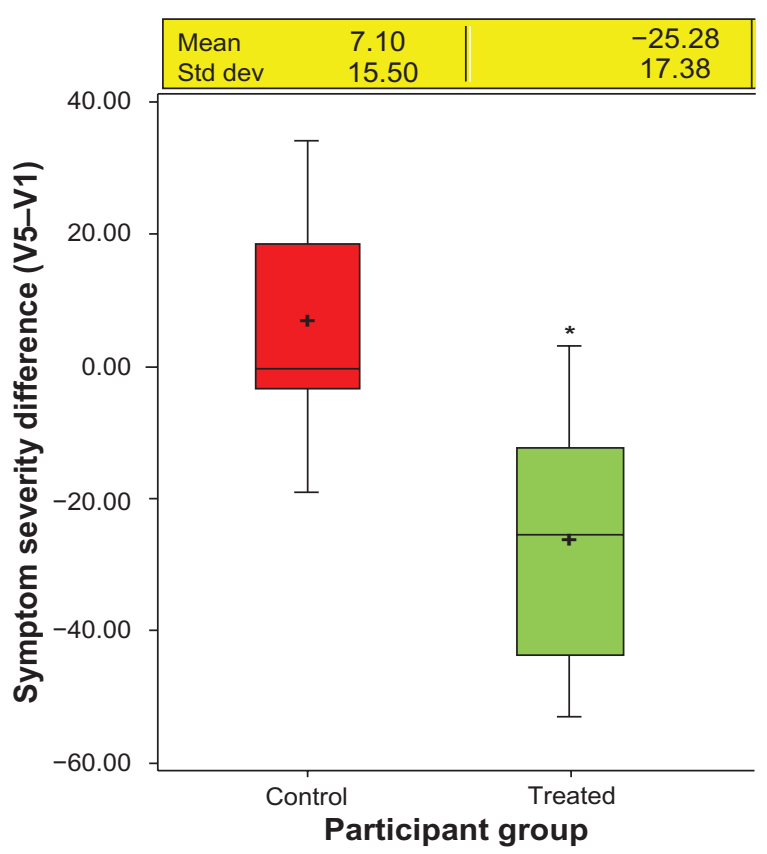

Figure 3 Epigallocatechin gallate (EGCG) treatment decreases symptom-severity (SS) score in patients with uterine fibroids. SS score dramatically decreased with 4 months' (visit 5 [V5] score < visit I [VI] score) use of green tea extract (EGCG, oral dose, $800 \mathrm{mg} /$ day). The mean change in SS score for the EGCG-treated group was $-25.28( \pm 17.38)$ and significantly low $(t=5.22, P<0.000 \mathrm{I})$ compared to the placebo group, for which mean SS score was $=7.1( \pm 15.5)$. A $P$-value of $\leq 0.05$ was considered statistically significant and is indicated by the asterisk. The box plot shows the maximum and minimum values as well as the mean and the median. Mean scores are indicated by plus signs. 
increase of $20.7 \pm 21.1$ in the percentile scores for HRQL (Figure 4). On the other hand, in the placebo group, the overall average (Figure 4) improvement in percentile score was only $2.19 \pm 17.4$. At the end of the 4-month treatment period, HRQL improved significantly in the EGCG treatment group $(P=0.017$, Figure 4$)$ compared to the placebo group.

\section{Visit-to-visit trends of SS and HRQL in response to EGCG treatment in patients with uterine fibroids}

The SS and HRQL scores were obtained from all patients at every visit (visit 1 to visit 5) during the 4-month study period. For each patient, the total scores for SS and HRQL were calculated as the percentage of maximum total scores possible for each questionnaire. Unlike the placebo, (Figure 5A and C), oral dose ( $800 \mathrm{mg} /$ day) EGCG treatment (Figure 5B and D) dramatically decreased SS scores, while HRQL scores increased with every visit during the treatment period.

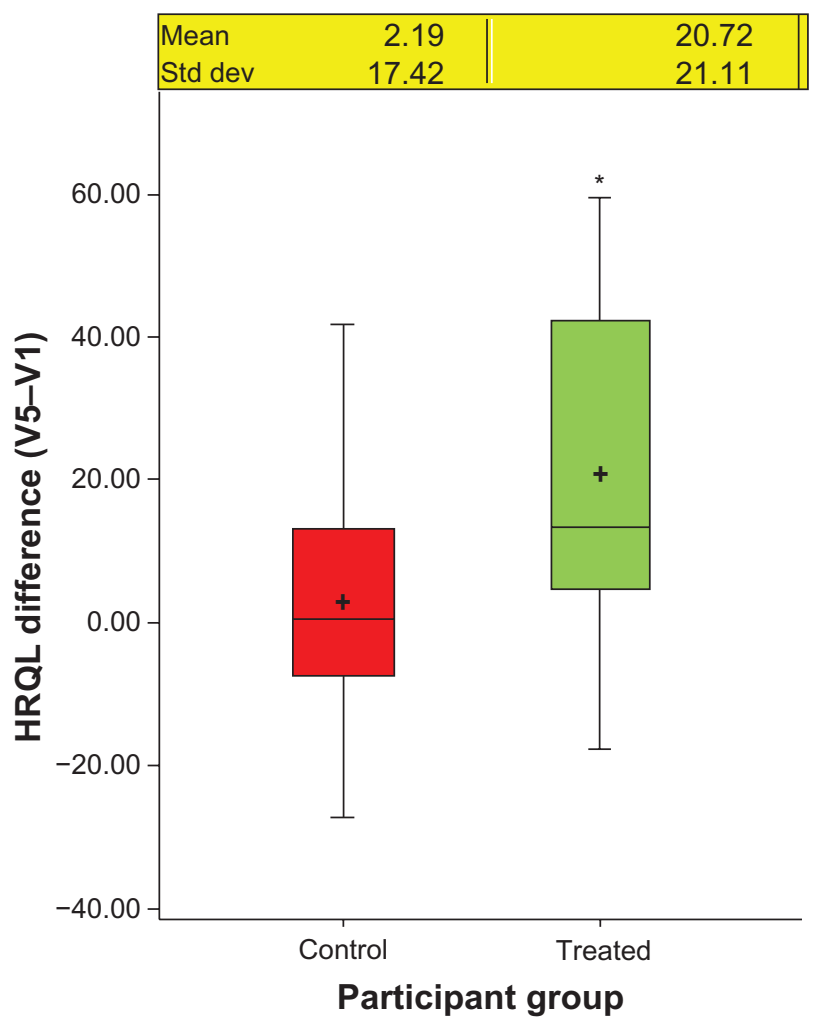

Figure 4 Epigallocatechin gallate (EGCG) treatment increases health-related quality-of-life (HRQL) score in patients with uterine fibroids. HRQL score increased dramatically with 4 months' (visit 5 [V5] score > visit I [VI] score) use of green tea extract (EGCG, oral dose, $800 \mathrm{mg} /$ day). The mean increase in HRQL in the EGCGtreated group was $20.72( \pm 2 \mathrm{I} . \mathrm{II})$ and significantly high $(t=1.25, P=0.0 \mathrm{I})$ compared to the placebo group, for which the mean percentile increase in HRQL was only 2.19 $( \pm 17.42)$. A $P$-value of $\leq 0.05$ was considered statistically significant and is indicated by the asterisk. The box plot shows the maximum and minimum values as well as the mean and the median. Mean scores are indicated by plus signs.
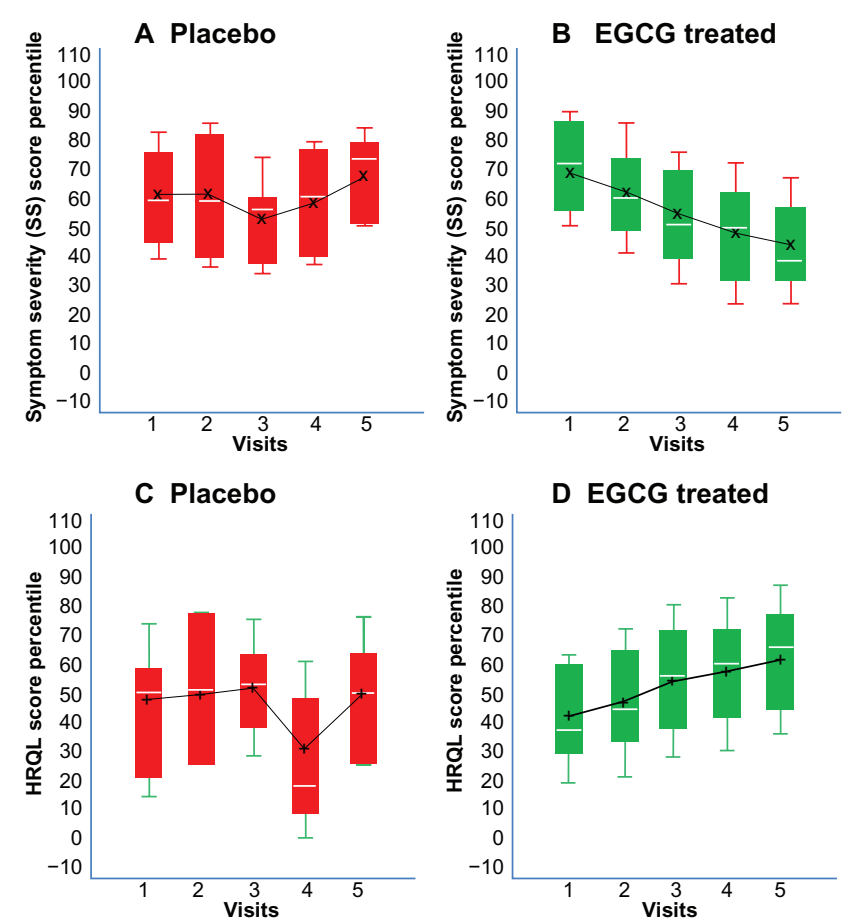

Figure 5 (A-D) Visit-to-visit quality-of-life improvement trends in response to epigallocatechin gallate (EGCG) treatment in patients with uterine fibroids. Oral dose $(800 \mathrm{mg} /$ day) of EGCG treatment dramatically increased health related qualityof-life (HRQL) score with every visit during the treatment period (visit I to visit 5 , 4 months) while symptom severity scores decreased. The box plots show the 25 th and 75th percentiles as well as the median, mean, and standard deviation ( \pm standard deviation). Mean scores are indicated by plus signs.

The SS scores showed a steady decline every month (69 at visit 1 to 44 at visit 5) in the EGCG-treated group (Figure 5B). The $\mathrm{SS}$ scores of women receiving placebo (Figure 5A) worsened from an average percentile score of 60 at baseline (visit 1) to 67 at the end of the study (visit 5). On the contrary, HRQL scores improved in the EGCG-treated group (Figure 5D), and the scores showed a steady incremental change at every visit (42 at visit 1 to 62 at visit 5). Interestingly, while the SS and HRQL evaluation of the EGCG-treated group showed a steady visit-to-visit trend, the changes in the placebo group (Figure 5A and $\mathrm{C}$ ) were erratic and not consistent from the beginning to the end of the study period.

\section{Effect of green tea extract (EGCG) treatment on menstrual bleeding pattern and blood loss in patients with uterine fibroids}

Using green tea extract (EGCG) was associated with significant improvements in the menstrual bleeding pattern among women with symptomatic UFs. Mean blood loss was assessed by patients every month using a pictogram 
questionnaire, ${ }^{25}$ as described in the Materials and methods section. By the end of 4 months, we had identified a significant decline in the average blood loss between the first and last visits in the treatment group (a decrease from $71 \mathrm{~mL} / \mathrm{month}$ to $45 \mathrm{~mL} / \mathrm{month}, P=0.001$, Table 2 ). On the other hand, in the placebo group, there was no statistical difference between monthly average menstrual blood loss during the study period $(P=0.83)$. Interestingly, three out of $22(14 \%)$ women in the treatment group developed amenorrhea in spite of normal serum FSH levels after 4 months of treatment, compared with none of the women in the placebo group. Treatment with green tea extract also had a significant effect on mean hemoglobin levels (EGCG vs placebo, $P=0.05)$. Mean hemoglobin levels increased in the treatment group from 11.7 to $12.4 \mathrm{~g} / \mathrm{dL}(P=0.02)$, while levels decreased in the placebo group from 12.95 to $12.5 \mathrm{~g} / \mathrm{dL}(P=0.03)$.

\section{Green tea extract (EGCG) treatment is well tolerated with favorable side-effect profile in subjects with symptomatic uterine fibroids}

None of the participants in either arm of this study noticed any adverse events of any grade throughout the whole study period. There were no abnormalities in liver-function tests, FSH level, or estradiol in any participants, and all inflammatory markers tested were within normal levels. No endometrial hyperplasia or endometrial pathology was detected during the study. Participants informed us that they tolerated the medication well.

\section{Discussion}

The current management options for uterine leiomyoma are typically individualized based on the severity of the symptoms, the size and location of the fibroid lesions, the patient's age, their chronological proximity to menopause, and the patient's desire for future fertility. The treatment options range from conservative measures (reassurance/ observation) to radical procedures (hysterectomy). ${ }^{34}$ Currently, there are no definitive FDA-approved agents for long-term medical treatment of uterine leiomyoma, as there is a remarkable lack of randomized trial data demonstrating the effectiveness of medical therapies. ${ }^{35}$ However, there are several candidate agents like GnRH analogues (leuprolide and goserelin), progesterone receptor antagonists (mifepristone), and more recently selective progesterone-receptor modulators such as ulipristal that have been shown to reduce uterine leiomyoma volume, reduce heavy menstrual bleeding, and restore hemoglobin levels in limited preliminary clinical studies. ${ }^{36-43}$ Recent findings suggest that volume maintenance and growth of human uterine leiomyomas are heavily progesterone dependent, and hence antiprogesterone could reverse leiomyoma growth effects. Clinical findings also support these laboratory observations: studies have involved the evaluation of mifepristone (RU 486), asoprisnil, and more recently CDB-2914 and CDB-4124 (CDB, Contraceptive Development Branch). ${ }^{8-10}$ However, none of these candidates has been approved for long-term management of uterine leiomyoma due to increased sideeffect risks, including pseudomenopausal symptoms, bone-density loss, and endometrial changes, including hyperplasia and cystic glandular changes. ${ }^{43-45}$

The aim of our study was to evaluate an oral regimen of green tea extract (EGCG) for treatment of uterine leiomyoma to avoid the aforementioned unfavorable side effects and possible serious complications. In our randomized, doubleblinded, placebo-controlled pilot study, $800 \mathrm{mg}$ of green tea extract was orally administered daily for 4 months to women with symptomatic fibroids confirmed by ultrasonography. After analyzing the data, we focused on: (1) change in total UF volume; monthly mean change in quality of life,

Table 2 Effect of green tea extract (epigallocatechin gallate [EGCG]) treatment on blood loss and hemoglobin levels in patients with uterine fibroids

\begin{tabular}{|c|c|c|c|c|c|}
\hline & $\begin{array}{l}\text { Treatment } \\
\text { Group }\end{array}$ & $\begin{array}{l}\text { Visit I } \\
\text { (before treatment) }\end{array}$ & $\begin{array}{l}\text { Visit } 5 \\
\text { (posttreatment) }\end{array}$ & $\begin{array}{l}\text { Change } \\
\text { (V5-VI) }\end{array}$ & $P$-value \\
\hline \multirow[t]{2}{*}{ Blood loss (mL/month) } & Placebo $(n=I I)$ & $72 \pm 33.11$ & $88 \pm 41.77$ & Increase & 0.083 (NS) \\
\hline & EGCG $(n=22)$ & $71.26 \pm 37.14$ & $45 \pm 27.91$ & Decrease & $0.00 I^{*}$ \\
\hline \multirow[t]{2}{*}{ Hemoglobin $(\mathrm{g} / \mathrm{dL})$} & Placebo $(\mathrm{n}=\mathrm{II})$ & $12.95 \pm 2.93$ & $12.5 \pm 2.39$ & Decrease & $0.03 *$ \\
\hline & EGCG $(n=22)$ & $|1.7 \pm 4.1|$ & $12.4 \pm 2.73$ & Increase & $0.02 *$ \\
\hline
\end{tabular}

Notes: Blood loss was assessed monthly by means of a daily menstrual log, with visual assessment of quantity of blood lost using a menstrual pictogram. The scores (in milliliters) were associated with each icon given in a pictogram that was provided to all participants. The pictograms were assessed monthly, along with safety monitoring, which included monthly evaluation of hemoglobin levels in blood samples collected at each visit (I-5). $P$-values of $\leq 0.05$ were considered statistically significant. *Statistically significant.

Abbreviation: NS, not significant. 
consisting of (2) assessment of fibroid-specific SS and (3) perceived impact of leiomyoma symptoms on HRQL; and (4) monthly blood-loss assessment (menstrual blood loss and hemoglobin level).

The overall UF volumes were compared at baseline and at the end of the 4-month treatment period. The overall percentage reduction in mean total fibroid volume due to EGCG treatment was highly significant compared to placebo treatment. Interestingly, while the placebo group showed increase in total fibroid volume, none of the participants in the EGCG treatment group showed any increase in fibroid volume. Only one showed no change, and all others showed shrinkage in fibroid volume. Such a volume increase in the placebo group is consistent also with other longitudinal observation studies of the natural history of uterine leiomyomata. ${ }^{46,47}$ At 4 months, the overall uterine volume also decreased by $18.7 \%$ in the EGCG treatment group compared to an increase by $14.65 \%$ in the placebo group. This range of changes in uterine volume is fairly similar to results obtained in ulipristal, goserelin, and leuprolide studies. ${ }^{36,38,43}$ The Carbonell Esteve et al study with mifepristone, however, did not show significant decrease in uterine volume in either treatment groups. ${ }^{48}$

There was a significant decline in the severity of symptoms in the EGCG treatment group compared to the placebo group; this was similar to the leuprolide, mifepristone, and ulipristal studies. ${ }^{38,43,49}$ Concurrently, we observed a significant increase in the leiomyoma-specific HRQL measures in the EGCG treatment group compared to the placebo group. However, $\mathrm{SS}$ worsened in the placebo group, while there was no significant change in their leiomyoma-specific quality of life at the end of the study, with only a $2 \%$ improvement, possibly due to the placebo effect or participant's subjective bias. ${ }^{50,51}$ The only dropouts were from the placebo group. It is very plausible that they were not relieved of symptoms and opted to seek other modalities of treatment. The improvement in HRQL and decline in SS in response to EGCG treatment was comparable to a study conducted by Hirzel et $\mathrm{al}^{52}$ to see the impact of uterine artery embolization on quality of life among symptomatic fibroid patients. The advantage of this oral intervention, however, would be the avoidance of possible side effects of uterine artery embolization, such as uterine necrosis and increased rate of surgical reintervention. ${ }^{53,54}$ We attribute the fibroid-shrinking effect of EGCG to the inhibitory effect on proliferation of leiomyoma tumor cells and induction of apoptosis, as we have shown in our recent preclinical work. ${ }^{11,18}$ There was no change in inflammatory markers, suggesting that the anti-inflammatory effect of
EGCG is not the primary mechanism behind declining SS, at least not at the global systemic level.

Our limited subject population was observed to have borderline anemia, with an average hemoglobin level at $11.7 \mathrm{~g} / \mathrm{dL}$, and using iron supplements was not an exclusion criterion for our study.

However, there was a significant rise $(P=0.02)$ reaching normal ranges $(12.1-15.1 \mathrm{~g} / \mathrm{dL})^{40}$ from baseline to the end of the study period within the treatment group and a significant difference when compared to the change in placebo group $(P<0.05)$. This objective biomarker also appropriately validates the subjects' reporting of decreased SS in the HRQL questionnaire.

Our study did not show any abnormalities in liver or renal functions. This is in agreement with Shen et al, ${ }^{55}$ where they observed that the levels of serum aspartate aminotransferase and alanine aminotransferase (both indicators of liver function), and serum blood urea nitrogen and creatinine (both indicators of renal function) were not affected with $500 \mathrm{mg}$ daily dose of green tea polyphenol supplementation for a period of 24 weeks. This observation was also reported in the Matsuyama et al study, where 24 weeks of daily beverage ingestion of $576 \mathrm{mg}$ did not raise any safety concerns in obese Japanese participants. ${ }^{56}$ The wide safety therapeutic margin of green tea extracts was also reported in several other recent reports. ${ }^{57-59}$ The Pisters et al study, ${ }^{60}$ with 49 adult cancer patients who used green tea extract (with $7 \%$ caffeine), encountered mild-to-moderate toxicities in the form of gastrointestinal disturbance (sore throat, nausea, and abdominal bloating), neurologic (insomnia, paresthesia, restlessness), and cardiovascular (palpitations) complaints. In addition, the constitutional complaints of diaphoresis and fatigue were also seen. The dose-limiting component in the green tea extracts reported by Pisters et al appears to be the caffeine, ${ }^{60}$ which was not present in the green tea supplements used in our trial. The fact that no adverse effects have been reported in our study confirms that decaffeinated green tea extract, at least the dosage used here, is safe and tolerable in reproductive-age women, as reflected by the low dropout rate. Furthermore, there was no endometrial hyperplasia or any endometrial abnormalities noted in our study, contrary to observations noted in prior studies using such agents as selective progesterone-receptor modulators or mifepristone. ${ }^{43,61}$ The limitations of our clinical trial include the small study sample, despite our intensive recruitment efforts, and thus results from our study warrant replication through a larger multicenter study.

This study, however, clearly demonstrates that green tea extract (EGCG) has a significant positive effect on 
the reduction of UF burden and SS. Our data demonstrate that subjects who used green tea extracts for 4 months experienced significant shrinkage in their total fibroid volume, significant reduction in SS, and significant consistent improvement in their quality of life. Thus, administration of green tea extract could possibly be an effective oral treatment for UFs. Our data suggest a promising alternative in which the burden of this disease could be reduced by making green tea extract available to women who have UFs. If confirmed in larger multicenter trials, this may provide a positive therapeutic and possibly preventive option for leiomyoma, especially in African-American women, who are particularly affected by this disease and are also avid users of alternative and complementary medicine. ${ }^{62}$ This novel noninvasive intervention for possible treatment and/ or prevention of UFs can have major positive impact on the reproductive health of women of all ethnic groups around the world.

\section{Acknowledgment}

This work was supported by grant 1 R01 HD046228-01 from the National Institute of Child Health and Human Development, National Institutes of Health and RCMI grant 2 G12 RR003032 to Dr Al-Hendy.

\section{Disclosure}

The authors report no conflicts of interest in this work.

\section{References}

1. Merrill RM. Hysterectomy surveillance in the United States, 1997 through 2005. Med Sci Monit. 2008;14:CR24-CR31.

2. Baird DD, Dunson DB, Hill MC, Cousins D, Schecctman JM. High cumulative incidence of uterine leiomyoma in black and white women: ultrasound evidence. Am J Obstet Gynecol. 2003;188:100-107.

3. Shen SH, Fennessy F, McDannold N, Jolesz F, Tempany C. Imageguided thermal therapy of uterine fibroids. Semin Ultrasound CT MR. 2009;30:91-104.

4. Othman EE, Al-Hendy A. Molecular genetics and racial disparities of uterine leiomyomas. Best Pract Res Clin Obstet Gynaecol. 2008;22: 589-601.

5. Al-Hendy A, Salama S. Gene therapy and uterine leiomyoma: a review. Hum Reprod Update. 2006;12:385-400.

6. Ezzati M, Norian JM, Segars JH. Management of uterine fibroids in the patient pursuing assisted reproductive technologies. Womens Health (Lond Engl). 2009;5:413-421.

7. Hassan MH, Othman EE, Hornung D, Al-Hendy A. Gene therapy of benign gynecological diseases. Adv Drug Deliv Rev. 2009;61:822-835.

8. Attardi BJ, Burgenson J, Hild SA, Reel JR. In vitro antiprogestational/ antiglucocorticoid activity and progestin and glucocorticoid receptor binding of the putative metabolites and synthetic derivatives of CDB2914, CDB-4124, and mifepristone. J Steroid Biochem Mol Biol. 2004:88:277-288

9. Williams AR, Critchley HO, Osei J, et al. The effects of the selective progesterone receptor modulator asoprisnil on the morphology of uterine tissues after 3 months treatment in patients with symptomatic uterine leiomyomata. Hum Reprod. 2007;22:1696-1704.
10. Yoshida S, Ohara N, Xu Q, et al. Cell-type specific actions of progesterone receptor modulators in the regulation of uterine leiomyoma growth. Semin Reprod Med. 2010;28:260-273.

11. Zhang D, Al-Hendy M, Richard-Davis G, Montgomery-Rice V, Rajaratnam V, Al-Hendy A. Antiproliferative and proapoptotic effects of epigallocatechin gallate on human leiomyoma cells. Fertil Steril. 2010;94:1887-1893.

12. US Department of Agriculture. USDA database for the flavonoid content of selected foods: release 2.1. 2007. Available from: http:// www.ars.usda.gov/SP2UserFiles/Place/12354500/Data/Flav/Flav02-1. pdf. Accessed May 10, 2013.

13. Khan N, Afag F, Saleem M, Ahmad N, Mukhtar H. Targeting multiple signaling pathways by green tea polyphenol (-)-epigallocatechin-3gallete. Cancer Res. 2006;66:2500-2505.

14. Wolfram S. Effects of green tea and EGCG on cardiovascular and metabolic health. J Am Coll Nutr. 2007;26:373S-388S.

15. Ahn WS, Yoo J, Huh SW, et al. Protective effects of green tea extracts (polyphenon E and EGCG) on human cervical lesions. Eur J Cancer Prev. 2003;12:383-390.

16. Chow HH, Hakim IA, Vining DR, et al. Effects of dosing condition on the oral bioavailability of green tea catechins after single-dose administration of polyphenon E in healthy individuals. Clin Cancer Res. 2005;11:4627-4633.

17. Luo H, Tang L, Tang M, et al. Phase IIa chemoprevention trial of green tea polyphenols in high-risk individuals of liver cancer: modulation of urinary excretion of green tea polyphenols and 8-hydroxydeoxyguanosine. Carcinogenesis. 2006;27:262-268.

18. Zhang D, Al-Hendy M, Richard-Davis G, et al. Green tea extract inhibits proliferation of uterine leiomyoma cells in vitro and in nude mice. Am J Obstet Gynecol. 2010;202:e1-e9.

19. Al-Hendy A, Salama SA. Catechol-O-methyltransferase polymorphism is associated with increased uterine leiomyoma risk in different ethnic groups. J Soc Gynecol Investig. 2006;13:136-144.

20. Lu H, Meng X, Yang CS. Enzymology of methylation of tea catechins and inhibition of catechol-O-methyltransferase by (-)-epigallocatechin gallate. Drug Metab Dispos. 2003;31:572-579.

21. Chen D, Wang CY, Lambert JD, Ai N, Welsh WJ, Yang CS. Inhibition of human liver catechol-O-methyltransferase by tea catechins and their metabolites: structure-activity relationship and molecular-modeling studies. Biochem Pharmocol. 2005;69:1523-1531.

22. Shixian Q, VanCrey B, Shi J, Kakuda Y, Jiang Y. Green tea extract thermogenesis-induced weight loss by epigallocatechin gallate inhibition of catechol-O-methyltransferase. J Med Food. 2006;9:451-458.

23. Salama SA, Ho SL, Wang HQ, Tenhunen J, Tilgmann C, Al-Hendy A. Hormonal regulation of catechol-O-methyl transferase activity in women with uterine leiomyomas. Fertil Steril. 2006;86:259-262.

24. Spies JB, Coyne K, Guaou Guaou N, Boyle D, Skyrnarz-Murphy K, Gonzalves SM. The UFS-QOL, a new disease-specific symptom and health-related quality of life questionnaire for leiomyomata. Obstet Gynecol. 2002;99:290-300.

25. Wyatt KM, Dimmock PW, Walker TJ, O'Brien PM. Determination of total menstruation blood loss. Fertil Steril. 2001;76:125-131.

26. Nair S, Al-Hendy A. Adipocytes enhance the proliferation of human leiomyoma cells via TNF- $\alpha$ proinflammatory cytokine. Reprod Sci. 2011;18:1186-1192.

27. Serafini M, Del Rio D, Yao DN, Bettuzzi S, Peluso I. Health benefits of tea. In: Benzie IFF, Wachtel-Galor S, editors. Herbal Medicine: Biomolecular and Clinical Aspects. 2nd ed. Boca Raton (FL): CRC Press; 2011. Chapter 12.

28. Han SG, Han SS, Toborek M, Hennig B. EGCG protects endothelial cells against PCB 126-induced inflammation through inhibition of AhR and induction of Nrf2-regulated genes. Toxicol Appl Pharmacol. 2012;261:181-188.

29. Hosokawa Y, Hosokawa I, Ozaki K, Nakanishi T, Nakae H, Matsuo T. Tea polyphenols inhibit IL-6 production in tumor necrosis factor superfamily 14 -stimulated human gingival fibroblasts. Mol Nutr Food Res. 2010;54:S151-S158. 
30. Jiang J, Mo ZC, Yin K, et al. Epigallocatechin-3-gallate prevents TNF-alpha-induced NF-kappaB activation thereby upregulating ABCA1 via the Nrf2/Keap1 pathway in macrophage foam cells. Int $J$ Mol Med. 2012;29:946-956.

31. Loke WM, Proudfoot JM, Hodgson JM, et al. Specific dietary polyphenols attenuate atherosclerosis in apolipoprotein E-knockout mice by alleviating inflammation and endothelial dysfunction. Arterioscler Thromb Vasc Biol. 2010;30:749-757.

32. Ozdemir S, Celik C, Gezginc K, Kiresi D, Esen H. Evaluation of endometrial thickness with transvaginal ultrasonography and histopathology in premenopausal women with abnormal vaginal bleeding. Arch Gynecol Obstet. 2010;282:395-399.

33. Maleki Z, Kim HS, Thonse VR, Judson K, Vinh TN, Vang R. Uterine artery embolization with trisacryl gelatin microspheres in women treated for leiomyomas: a clinicopathologic analysis of alterations in gynecologic surgical specimens. Int J Gynecol Pathol. 2010;29:260-268.

34. Zhang Y, Peng W, Clarke J, Liu Z. Acupuncture for uterine fibroids. Cochrane Database Syst Rev. 2010;1:CD007221.

35. Viswanathan M, Hartmann K, McKoy N, et al. Management of uterine fibroids: an update of the evidence. Evid Rep Technol Assess (Full Rep). 2007;154:1-122.

36. Donnez J, Hervais Vivancos B, Kudela M, Audebert A, Jadoul P. A randomized, placebo-controlled, dose-ranging trial comparing fulvestrant with goserelin in premenopausal patients with uterine fibroids awaiting hysterectomy. Fertil Steril. 2003;79:1380-1389.

37. Deligdisch L, Hirschmann S, Altchek A. Pathologic changes in gonadotropin releasing hormone agonist analogue treated uterine leiomyomata. Fertil Steril. 1997;67:837-841.

38. Friedman AJ, Hoffman DI, Comite F, Browneller RW, Miller JD. Treatment of leiomyomata uteri with leuprolide acetate depot: a doubleblind, placebo-controlled, multicenter study. The Leuprolide Study Group. Obstet Gynecol. 1991;77:720-725.

39. Khan KN, Kitajima M, Hiraki K, et al. Changes in tissue inflammation, angiogenesis and apoptosis in endometriosis, adenomyosis and uterine myoma after GnRH agonist therapy. Hum Reprod. 2010;25:642-653.

40. Palomba S, Russo T, Orio F Jr, et al. Effectiveness of combined GnRH analogue plus raloxifene administration in the treatment of uterine leiomyomas: a prospective, randomized, single-blind, placebo-controlled clinical trial. Hum Reprod. 2002;17:3213-3219.

41. Steinauer J, Pritts EA, Jackson R, Jacoby AF. Systematic review of mifepristone for the treatment of uterine leiomyomata. Obstet Gynecol. 2004;103:1331-1336.

42. Donnez J, Tomaszewski J, Vasquez F, et al. Ulipristal acetate versus leuprolide acetate for uterine fibroids. N Engl J Med. 2012;366:421-432.

43. Donnez J, Tatarchuk TF, Bouchard P, et al. Ulipristal acetate versus placebo for fibroid treatment before surgery. $N$ Engl J Med. 2012;366: 409-420.

44. Surrey ES. Gonadotropin-releasing hormone agonist and add-back therapy: what do the data show? Curr Opin Obstet Gynecol. 2010;22: 283-238.

45. Spitz IM. Clinical utility of progesterone receptor modulators and their effect on the endometrium. Curr Opin Obstet Gynecol. 2009;21: 318-324.

46. Mavrelos D, Ben-Nagi J, Holland T, Hoo W, Naftalin J, Jurkovic D. The natural history of fibroids. Ultrasound Obstet Gynecol. 2010;35: $238-242$.
47. Bukulmez O, Doody KJ. Clinical features of myomas. Obstet Gynecol Clin North Am. 2006;33:69-84.

48. Carbonell Esteve JL, Riverón AM, Cano M, et al. Mifepristone $2.5 \mathrm{mg}$ versus $5 \mathrm{mg}$ daily in the treatment of leiomyoma before surgery. Int J Womens Health. 2012;4:75-84.

49. Fiscella K, Eisinger SH, Meldrum S, Feng C, Fisher SG, Guzick DS. Effect of mifepristone for symptomatic leiomyomata on quality of life and uterine size: a randomized controlled trial. Obstet Gynecol. 2006;108:1381-1387.

50. Scott DJ, Stohler CS, Egnatuk CM, Wang H, Koeppe RA, Zubieta JK. Placebo and nocebo effects are defined by opposite opioid and dopaminergic responses. Arch Gen Psychiatry. 2008;65: 220-231.

51. Finniss DG, Kaptchuk TJ, Miller F, Benedetti F. Biological, clinical, and ethical advances of placebo effects. Lancet. 2010;375:686-695.

52. Hirzel F, Hess T, Zollikofer C. Quality control and quality of life after uterine fibroid embolization: long-term results. Gynakol Geburtshilfliche Rundsch. 2008;48:84-93. German

53. Maheux-Lacroix S, Lemyre M, Laberge PY, Lamarre A, Bujold E. Uterine artery embolization complicated by uterine perforation at the site of previous myomectomy. J Minim Invasive Gynecol. 2012;19: $128-130$.

54. Gupta JK, Sinha A, Lumsden MA, Hickey M. Uterine artery embolization for symptomatic uterine fibroids. Cochrane Database Syst Rev. 2012;16:CD005073.

55. Shen CL, Chyu MC, Pence BC, Yeh JK, Zhang Y, Felton CK. Green tea polyphenols supplementation and tai chi exercise for postmenopausal osteopenic women: safety and quality of life report. BMC Complement Altern Med. 2010;10:76.

56. Matsuyama T, Tanaka Y, Kamimaki I, Nagao T, Taokimitsu I. Catechin safely improved higher levels of fatness, blood pressure, and cholesterol in children. Obesity (Silver Spring). 2008;16:1338-1348.

57. Fiorini RN, Donovan JL, Rodwell D, et al. Short-term administration of (-)-epigallocatechin gallate reduces hepatic steatosis and protects against warm hepatic ischemia/reperfusion injury in steatotic mice. Liver Transpl. 2005;11:298-308.

58. Nishikawa T, Nakajimi T, Moriguchi M, et al. A green tea polyphenol, epigalocatechin-3-gallate, induces apoptosis of human hepatocellular carcinoma, possibly through inhibition of Bcl-2 family proteins. J Hepatol. 2006;44:1074-1082.

59. Bruno RS, Dugan CE, Smyth JA, DiNatale DA, Koo SI. Green tea extract protects leptin-deficient, spontaneously obese mice from hepatic steatosis and injury. $J$ Nutr. 2008;138:323-331.

60. Pisters KM, Newman RA, Coldman B, Shin DM, Khuri FR, Hong WK. Phase I trial of oral green tea extract in adult patients with solid tumors. J Clin Oncol. 2001;19:1830-1838.

61. Engman M, Granberg S, Williams AR, Meng CX, Lalitkumar PG, Gemzell-Danielsson K. Mifepristone for treatment of uterine leiomyoma. A prospective randomized placebo controlled trial. Hum Reprod. 2009;24:1870-1879.

62. Chao MT, Wade CM. Socioeconomic factors and women's use of complementary and alternative medicine in four racial/ethnic groups. Ethn Dis. 2008;18:65-71.
International Journal of Women's Health

\section{Publish your work in this journal}

The International Journal of Women's Health is an international, peerreviewed open-access journal publishing original research, reports, editorials, reviews and commentaries on all aspects of women's healthcare including gynecology, obstetrics, and breast cancer. The manuscript management system is completely online and includes
Dovepress

a very quick and fair peer-review system, which is all easy to use. Visit http://www.dovepress.com/testimonials.php to read real quotes from published authors. 